\title{
Exploring bridge dynamics for ultra-high-speed, Hyperloop, trains
}

\author{
Nicholas A Alexander ${ }^{1}$, Mohammad M Kashani ${ }^{2}$ \\ ${ }^{1}$ Senior Lecturer in structural engineering, University of Bristol \\ ${ }^{2}$ Lecturer in structural engineering, University of Southampton, and visiting lecturer in University of \\ Bristol
}

\begin{abstract}
In this paper the dynamics of a set of ultra-high-speed (UHS) moving masses/loads traversing a continuous beam are explored. The proposed model is intended to simulate the dynamic response of continues bridges under the new Hyperloop/Transpod trains, which are proposed to travel at up to $1200 \mathrm{~km} / \mathrm{h}$. This speed introduces a range of dynamic responses that have hitherto not been observed in generic high-speed trains. The analytical results show that the dynamic amplification factors, due to train passage, are significantly larger than current trains. This is due to the combination of ultra-highspeed and continuous beam construction, which is necessary to maintain a partial vacuum in the enclosed tube. Therefore, current design recommendations are not sufficient for these UHS trains.
\end{abstract}

\section{Introduction}

The Hyperloop Alpha [1, 2] and Transpod [3] are trains that travel at Ultra-High-Speeds (UHS trains). This UHS is achieved by having the train travel within a 'vacuum' tube as shown in figure 1. Traveling within these tubes, which are continuous beams, allows a train to circumvent the air resistance, drag forces, of conventional high-speed trains. In addition, this UHS conceptual design makes use of magnetic levitation and linear accelerators as a means of propulsion. Thus, the concept is to reduce friction, in all its forms to an extremely low level. The proposed working speed of around $970 \mathrm{~km} / \mathrm{h}$, with a top speed of $1,200 \mathrm{~km} / \mathrm{h}$ has been suggested. This compares with an average working speed of $270 \mathrm{~km} / \mathrm{h}$ for High-Speed trains (HS trains). The latest record for a conventional passenger train is held by an SNCF (France) TGV POS trainset, which reached $574.8 \mathrm{~km} / \mathrm{h}(357.2 \mathrm{mph})$ in 2007 . This speed was exceeded (in Japan on a national test track) by the unconventional seven-car L0 series trainset which attained a speed of $603 \mathrm{~km} / \mathrm{h}(375 \mathrm{mph})$ in 2015.

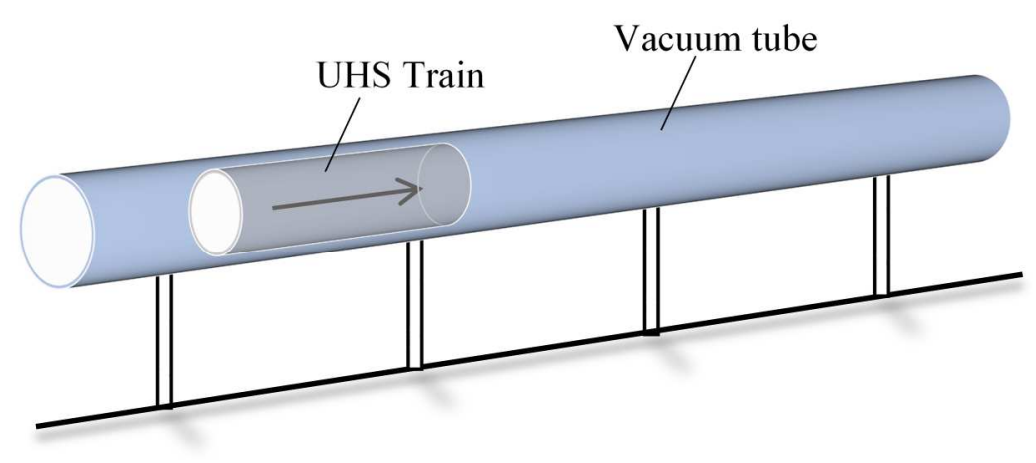

Figure 1: Initial conceptual design of prototype Hyperloop one. 
The current state-of-practice for the design and assessment of railway bridges in the UK is comprehensively treated in [4]. This document considers the effect of impact, oscillation and track and wheel irregularities. It suggests that bridge dynamics only plays an important role at train speeds above $160 \mathrm{~km} / \mathrm{h}$. Therefore, [4] recommends that the dynamic amplification factor (DAF) is 1 for train speeds below $160 \mathrm{~km} / \mathrm{h}$. This has been confirmed by other researchers in the assessment of existing railway bridges in the UK using nonlinear analysis techniques [5, 6]. Eurocode EN 1991-2 [7] has similar methods for calculation of the DAF for train speeds up to $200 \mathrm{~km} / \mathrm{h}$. However, [7] suggests more rigorous dynamic analysis is required to calculate the DAF for train speeds more than $200 \mathrm{~km} / \mathrm{h}$. Thus, for conventional HS trains, there is a need to consider the dynamic amplification effects by more thorough and bridge/train specific analyses. Nevertheless, this dynamic amplification while important is still likely to be relatively small at speeds of $270 \mathrm{~km} / \mathrm{h}$.

The UHS trains could travel at speeds more than four times the average speed of HS trains. This would be double the current world record speed. At these speeds the importance of dynamic amplification may be significant. This raises the new, currently unsolved, question of what is an appropriate DAF for this case. Furthermore, the Hyperloop/Transpod tubes will be supported by a series of piers which constrain the tube in the vertical direction but allow longitudinal slip for thermal expansion as well as dampened lateral slip to reduce the risk posed by earthquakes. The spacing of the Hyperloop piers retaining the tube is critical to achieve the design objective of the tube structure. The average spacing is $30 \mathrm{~m}$, which means there will be near 25,000 piers between the proposed San Francisco-Los Angeles line [1,2]. This imposes very large dynamic loading on the piers, which is currently not considered in any design standards. Therefore, exploring the impact of UHS train on the current DAF in the design standards is vital.

The mathematics of a moving force was first discussed by [8] and in the excellent and thorough treatise [9] that discusses both moving force and mass problems of simple spans. However, only a very limited class of simply moving load problems can be solved analytically. Thus, numerical methods are necessary for more general moving load simulations [10]. Authors [11] present a good historical review of the code based dynamic amplification factors (DAF) caused with travelling loads in the context of road traffic. The DAF represents the increase in quasi-static peak deflections and/or stress caused by the dynamics of the travelling load. A significant component of the DAF, in this case of highway traffic, is due to the impulsive 'shock' loads of heavy vehicles traversing defects in the road surface. The true moving mass/load dynamic amplification is very minimal at the speeds of highway traffic. Therefore, larger values of DAF are observed in the cases of high speed trains. A similar concept is also considered in railway bridges due to misalignment and defects of tracks. However, this is not currently considered an issue in UHS trains, as they float inside a vacuum tube with minimum friction. Therefore, the main parameters affecting the DAF in UHS is the resonance response of the structural system to UHS dynamic loading.

The theoretical and experimental study [12] elegantly transforms the problem of a moving force into the frequency domain however this cannot easily account for changes in system mass with time. Latterly many authors [13-17] seek to explore the dynamics of the bridge and sprung mass dynamics of the HS trains using finite element type formulations and experimental evidences. While these studies are important they are focused on very clearly defined engineering problems of specific trainsets traveling in relatively low speeds, across well-defined bridges. Thus, the problem of the dynamics of ultra-highspeed trains (UHS trains), such as the Hyperloop/Transpod, are unexplored to date.

The aim of this paper is to explore the likely envelope in the dynamic behaviour of these Hyperloop/Transpod UHS trains passing across continuous, multi-span, bridges of any span length. We derive the system equations of motion for this problem, using a 'tensorial' Rayleigh-Ritz type formulation. After identifying all the key non-dimensional groups, we perform a systematic parametric exploration of this problem. Finally, we propose a likely upper bound to the dynamic amplification 
factor (DAF) imposed on this class of bridges for a generic class of UHS trains. Furthermore, we seek to determine whether the current design code recommendations are suitable for such UHS trains.

\section{Theory}

In this section we derive, from first principles, the equations of motion of a train composed of a set of moving masses/loads traveling at any speed across a continuous beam of any span length with any number of spans. Consider a set $k$ moving masses $m_{p}$ (at positions $x_{p}$ ) traveling at some group velocity $v$ across a continuous beam of $n$ spans of length $L$; shown in the figure 2 . The beam has a uniform mass per unit length $m$ and flexural rigidity $E I$.

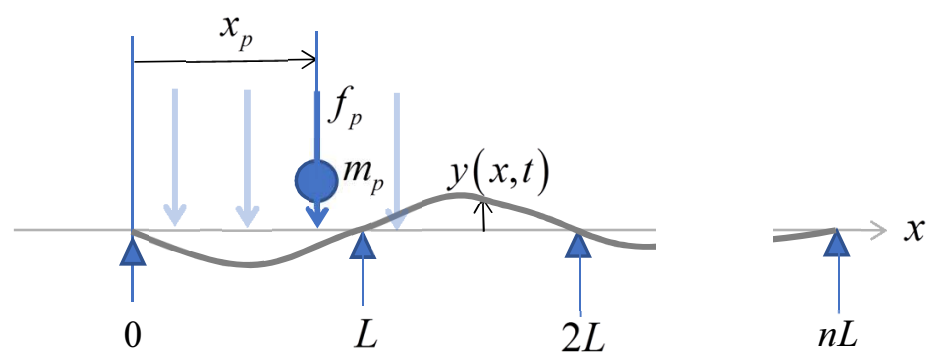

Figure 2, a train composed of set of $k$ moving point masses traveling across an $n$ span continuous beam

\subsection{Lagrangian formulation}

The kinetic energy $Q$ for this system is composed of two terms; (i) is due to the kinetic energy of the bridge and (ii) is due to the kinetic energy of the train. $Q$ is defined as follows

$$
Q=\frac{1}{2} m \int_{0}^{n L} \dot{y}^{2} \mathrm{~d} x+\frac{1}{2} \sum_{p=1}^{k}\left\{m_{p} \dot{y}\left(x_{p}, t\right)^{2} \beta\left(x_{p}\right)\right\},
$$

where $y(x, t)$ is the spatiotemporal beam displacement. In UHS trains, the moving masses $m_{i}$ would correspond to the locations of the magnetic levitation bearings. In conventional HS trains, this would correspond to the wheelset locations. The boxcar function $\beta(x)$ ensures only travelling masses 'on the beam' are included in the energy considerations. This boxcar function can be defined as follows in terms of Heaviside functions $H(x)$ thus,

$$
\beta(x)=H(x)-H(x-n L)
$$

To simplify the resulting equations of motion and to identify all the key non-dimensional parameter groups we introduce a non-dimensional coordinate $\xi$ where $x=\xi L$ and train moving mass positions are $x_{p}=\xi_{p} L$. Hence, equation (1) is re-stated as follows,

$$
Q=\frac{m L}{2} \int_{0}^{n} \dot{y}^{2} \mathrm{~d} \xi+\frac{1}{2} \sum_{p=1}^{k}\left\{m_{p} \dot{y}\left(\xi_{p}, t\right)^{2} \beta\left(\xi_{p}\right)\right\},
$$


where the beam displacement is now $y(\xi, t)$. Note that this change in coordinates $x=\xi L$ changes the integral limits to 0 to $n$.

The potential energy $V$ of the system is also composed of two terms; (i) is the internal flexural strain energy of the beam and (ii) is external work done in moving the gravitational load of the train. $V$ is defined as follows

$$
V=\frac{1}{2} E I \int_{0}^{n L}\left(\frac{\mathrm{d}^{2} y}{\mathrm{~d} x^{2}}\right)^{2} \mathrm{~d} x-\sum_{p=1}^{k}\left\{f_{p} y\left(x_{p}, t\right) \beta\left(x_{p}\right)\right\}
$$

where the gravitational train loads are $f_{p}=-m_{p} g$. As before we introduce non-dimensional coordinate $\xi$ where $x=\xi L$. Hence, beam curvature is redefined as follows, $\mathrm{d}^{2} y / \mathrm{d} x^{2}=\left(1 / L^{2}\right) \mathrm{d}^{2} y / \mathrm{d} \xi^{2}$. We use the Newtonian prime notation $(\bullet)^{\prime \prime}=\mathrm{d}^{2} y / \mathrm{d} \xi^{2}$ and hence equation (4) is re-expressed as

$$
V=\frac{E I}{2 L^{3}} \int_{0}^{n} y^{\prime \prime 2} \mathrm{~d} \xi-\sum_{p=1}^{k}\left\{f_{p} y\left(\xi_{p}, t\right) \beta\left(\xi_{p}\right)\right\}
$$

where the beam displacement is now $y(\xi, t)$. To use the minimum action principle (Euler-Lagrange equations of motion) we need to introduce a spatiotemporal expansion of the beam displacement of the following form,

$$
y(\xi, t)=\sum_{i=1}^{q}\left\{\phi_{i}(\xi) u_{i}(t)\right\}=\phi_{i} u_{i}
$$

where the spatial parts (shape functions) are defined by $\phi_{i}(\xi)$ and temporal parts are defined by the $q$ degrees of freedom $u_{i}(t)$. We may choose any functions $\phi_{i}(\xi)$ that satisfy the beam support boundary conditions. To ensure an accurate model of the system dynamics with a small number of dofs $q$ the basis $\phi_{i}(\xi)$ is ideally a good approximation of the mode shapes of the system.

In this expression we shall make use of tensorial notation (repeat suffixes imply summation in a product term $[18,19])$. Also note that in this paper suffixes $i$ and $j$ are natural numbers and are in the range 1 to $q$. And so we state the Lagrangian $\Pi$ (Kinetic minus potential energies) as follows

$$
\Pi=\frac{1}{2}\left(M_{i j}+N_{i j}\right) \dot{u}_{i} \dot{u}_{j}-\frac{1}{2} K_{i j} u_{i} u_{j}-\int F_{i} \mathrm{~d} u_{j},
$$

where $u_{i}$ are the system generalised coordinates (dofs) which are approximately modal amplitudes. The rank 2 tensors (Matrices) above are defined as follows

$$
\begin{aligned}
& M_{i j}=m L \int_{0}^{n} \phi_{i} \phi_{j} \mathrm{~d} \xi, \quad N_{i j}=\sum_{p=1}^{k}\left(\phi_{i}\left(\xi_{p}\right) \phi_{j}\left(\xi_{p}\right) \beta\left(\xi_{p}\right)\right) \\
& K_{i j}=\frac{E I}{L^{3}} \int_{0}^{n} \phi_{i}^{\prime \prime} \phi_{j}^{\prime \prime} \mathrm{d} \xi, \quad F_{j}=-\sum_{p=1}^{k}\left(m_{p} g \phi_{j}\left(\xi_{p}\right) \beta\left(\xi_{p}\right)\right)
\end{aligned}
$$

where $M_{i j}$ this bridge mass matrix, $N_{i j}$ is the time-varying mass matrix of the moving masses of the train, $K_{i j}$ is the bridge stiffness matrix and $F_{j}$ is the time-varying applied system loads. And so the Euler-Lagrange equation of motion is 


$$
\left(M_{i j}+N_{i j}\right) \ddot{u}_{i}+C_{i j} \dot{u}_{i}+K_{i j} u_{i}=F_{j},
$$

where a term $C_{i j} \dot{u}_{i}$ has also been added to model the energy loses due to damping. A good approximation to modal basis for a multi-span (with equal spans) continuous beam is a Fourier sin series,

$$
\phi_{i}=\sin (i \pi \xi)
$$

Hence, the beam mass matrix $M_{i j}$ and stiffness matrix $K_{i j}$ are defined as follows

$$
M_{i j}=\frac{1}{2} n m L \delta_{i j}, \quad K_{i j}=\left\{\begin{array}{ccc}
\frac{1}{2} n \pi^{4} i^{4} \frac{E I}{L^{3}} & : & i=j \\
0 & : & i \neq j
\end{array},\right.
$$

where $\delta_{i j}$ is the Kronecker delta tensor (that is tensorial form of the identity matrix). Hence the system natural circular frequencies are

$$
\omega_{i}^{2}=i^{4} \pi^{4} \frac{E I}{m L^{4}}
$$

If we assume $C_{i j}$ is an orthogonal damping matrix where the $j$ th modal ratio of critical damping is $\gamma_{j}$ then we can define $C_{i j}$ as follows

$$
C_{i j} \dot{u}_{i}=2\left\{\gamma_{j} \omega_{j}\right\} M_{i j} \dot{u}_{i}
$$

In this paper, terms within braces \{\} do not imply a tensorial summation (inside or outside of the brace). The traveling load mass matrix $N_{i j}$ and forcing vector $F_{j}$ are defined as follows;

$$
\begin{gathered}
N_{i j}=\sum_{p=1}^{k}\left(m_{p} \sin \left(i \pi \xi_{p}\right) \sin \left(j \pi \xi_{p}\right) \beta\left(\xi_{p}\right)\right), \\
F_{j}=-\sum_{p=1}^{k}\left(m_{p} g \sin \left(j \pi \xi_{p}\right) \beta\left(\xi_{p}\right)\right)
\end{gathered}
$$

\subsection{Non-dimensional form of equation of motion}

Finally, we seek to express equation (9) in a non-dimensional form, by scaling approximate modal amplitudes $u$ and time $t$.

$$
u=\frac{g}{\omega_{1}^{2}} z, \quad t=\frac{1}{\omega_{1}} \tau
$$

Additionally, we pre-multiply equation (9) by the inverse of beam mass matrix, thus

$$
\left(\delta_{i j}+N_{i j}^{*}\right) \ddot{z}_{i}+\left\{2 \gamma_{j} j^{2}\right\} \dot{z}_{j}+\left\{j^{4}\right\} z_{j}=F_{j}^{*},
$$

where the Newtonian dots $(\dot{\bullet})=\partial \bullet / \partial \tau$ now signify derivative with respect to normalised time $\tau$. Terms within braces such as $\left\{j^{4}\right\}$ are not tensorial summed with terms outside the brace. Hence, here the 
tensorial suffix $j$ is just raised to the power 4 . The term $N_{i j}^{*}$ that couples the equations of motion is the mass matrix of the traveling mass (of the train) normalised with respect to the beam mass matrix,

$$
N_{i j}^{*}=\frac{2}{n} \sum_{p=1}^{k}\left\{\alpha_{p} \sin \left(i \pi \xi_{p}\right) \sin \left(j \pi \xi_{p}\right) \beta\left(\xi_{p}\right)\right\},
$$

where $\alpha_{p}$ are the mass ratios of the $p^{\text {th }}$ traveling load/mass (wheel) to a beam mass of a single span, as follows

$$
\alpha_{p}=\frac{m_{p}}{m L}
$$

Additionally, the normalised forcing vector $F_{j}^{*}$ is defined as

$$
F_{j}^{*}=-\frac{2}{n} \sum_{p=1}^{k}\left\{\alpha_{k} \sin \left(j \pi \xi_{p}\right) \beta\left(\xi_{p}\right)\right\}
$$

In contrast, quasi-static analysis is performed by setting all damping and inertial terms in equations (17) to zero and hence we obtain the following

$$
z_{j}=\left\{j^{-4}\right\} F_{j}^{*}
$$

This equation (21) is still a time-dependant linear system. It is however, an algebraic rather than differential system.

\subsection{Non-dimensional moving load speed $\Omega$}

Finally we define the moving loads position in terms of its group velocity $v$ and its starting position, at $t=0$ is $s_{p} L$ with respect to the origin.

$$
\xi_{p}=\frac{s_{p} L+v t}{L}=s_{p}+\frac{v}{\omega_{1} L} \tau
$$

The non-dimensional position of the $p^{\text {th }}$ train load is defined by term $j \pi \xi_{p}$ which is used in the added mass term $N_{i j}^{*}$ (equations (18)) and added load term $F_{j}^{*}$ (equation (20)). This term $j \pi \xi_{p}$ can be reexpressed as $j(\Omega \tau+\theta)$, where the primary excitation frequency for the travelling load, for the mode $j$, is $j \Omega$ and phase angles $j \theta$. These are defined as follows

$$
\Omega=\frac{\pi v}{\omega_{1} L}, \quad \theta=\pi s_{p}
$$

Thus, the non-dimensional speed $\Omega$ is a key system parameter, and is a function of span length $L$, dimensional group velocity $v$ and the fundamental circular frequency of the bridge $\omega_{1}$.

Ref [20] suggested an empirical formula for the fundamental natural frequencies of highway bridges of different spans; while [4] suggests a low and upper bound to the fundamental natural frequency of railway bridges. Figure 3(a) display these empirical relationships for the fundamental natural frequency vs span for highway and railway bridges. These two graphs are not that significantly different given the observed scatter in the experimental dataset. The empirical relationship between span and frequency 
for a Hyperloop/Transpod railway bridges is not determined yet since these have not been constructed. Nevertheless we will employ the mean frequency in [4] in conjunction with equation (23) to produce Figure 2(b). This figure describes the approximate variation of non-dimensional velocity $\Omega$ vs moving load speed and span length for railway bridges and trains. The average high speed train achieves a value of non-dimensional speed range of $0<\Omega \leq 1 / 3$ regardless of span while Hyperloop/Transpod trains have a proposed range $0<\Omega \leq 4 / 3$.
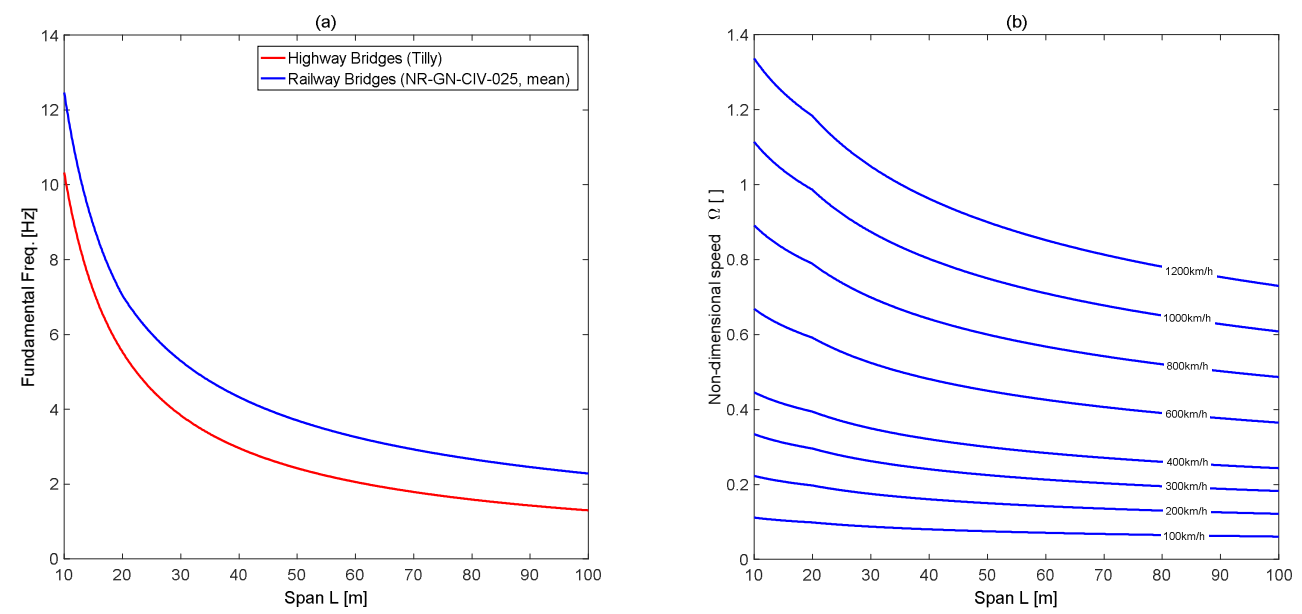

Figure 3, (a) variation of fundamental bridge frequency vs span length (b) variation of nondimensional velocity $\Omega$ with dimensional span and train speed for Railway Bridges.

\subsection{Dynamic amplification factor $\eta$}

We can define the dynamic amplification factor $\eta$ (DAF) as the maximum absolute deflection at any point on the multi-span beam (for a given load set travelling at a normalised speed $\Omega$ ) divided by the maximum deflection in the quasi-static case $(\Omega \simeq 0)$. Thus

$$
\eta=\frac{\max _{\tau, \xi}|y(\tau, \xi)|_{\text {dynamic }}}{\max _{\tau, \xi}|y(\tau, \xi)|_{\text {quasi-static }}}
$$

Therefore, determining the DAF for beam subject to a set of moving masses/loads requires the solution of dynamic equations (17) and quasi-static equations (21). The system parameters for problem are as follows

i. $\quad$ The normalised train speed $\Omega$

ii. The number of moving masses/loads $k$

iii. The total number of spans $n$ of length $L$ in the continuous beam

iv. The mass ratios $\alpha_{k}$ which are wheelset mass to beam single span mass.

v. The delays (phasing) of loads $s_{k}$ which depend on the carriage wheelset spacing

vi. The ratio of critical damping $\gamma_{j}$ for the $j$ th mode 


\section{Analysis and results}

\subsection{A single moving mass on multi-span beam}

The solutions of dynamic equations (17) and quasi-static equations (21), for the centre span location, are displayed in Figure 4(a). This is for system parameter $\Omega=0.2, \gamma_{i}=0.05, \alpha_{k}=0.1$. The horizontal axis is scaled time $\tau / \tau_{d}$ where $\tau_{d}=\omega_{1} L / v=\Omega / \pi$ is the non-dimensional time it takes for the moving mass to traverse the beam span $L$. Thus at $\tau / \tau_{d}=1$ the moving load has traversed the first span regardless of speed or span. The differences between quasi-static and dynamic solutions are apparent. The location and magnitude of the absolute dynamic amplification factors (DAF) are different. These graphs are comparable with specific results in ref [8-10]. Figure 4(b) displays the dynamic amplification factor, equation (24), which is the maximum absolute value at any point across the bridge (although this is normally close to the centre span).

Figure 5 shows that the effect of going from single span to multi-span cases, is the same as moving from (i) a transient dynamics dominated response problem to (ii) a steady-state resonance dominated problem. This figure is for the case of a single moving mass $(k=1)$ on a continuous beam. We observe an increase in the maximum DAF because the periodic loading is in contact with the beam for far more cycles of loading. Hence, as the number of spans increase resonant response behaviour dominates. These DAF functions asymptotically tend towards the classical amplification of $\eta \approx 1 / 2 \gamma$ for a single degree of freedom system subjected to sinusoidal excitation [20]. It is fortunate that the current maximum speed for high speed trains (HS train) is below this resonance, and that continuous spans do not in practice extend to large $n$ without some thermal expansion joints. Note that the average maximum speed of in service HS trains world-wide is about $270 \mathrm{~km} / \mathrm{h}$ and this corresponds approximately to a range of non-dimensional speed $0<\Omega \leq 1 / 3$ and this suggest a dynamic amplification factor, due to moving mass/load, is $\eta \leq 1.4$.

However, the Hyperloop/Transpod proposed train speed is fast enough to have to seriously consider the dynamic amplification effects of this resonance. Additionally, the need to maintain the vacuum might preclude the frequent use of expansion joints which would suggest a much larger number of spans $n$ this would induce more dynamic vulnerability.
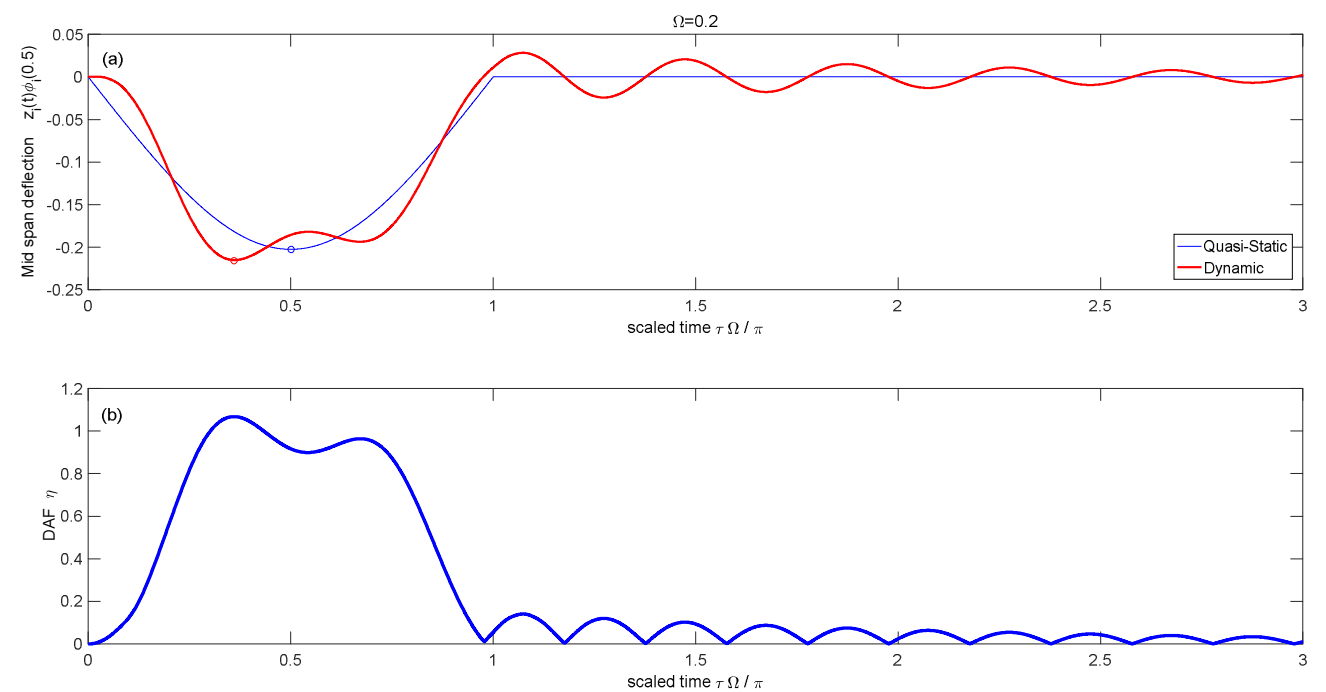

Figure 4, (a) Normalised midspan deflection (quasi-static, dynamic) vs time (b) Dynamic amplification factor vs time. 


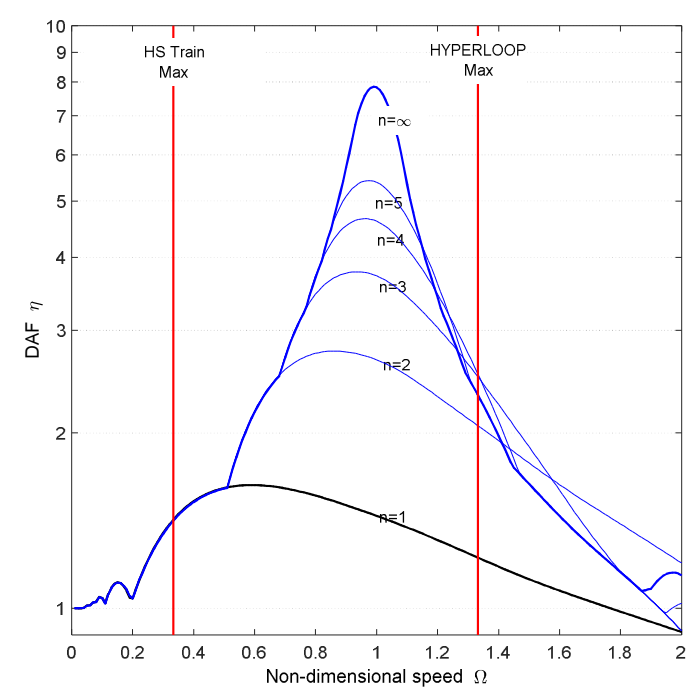

Figure 5. dynamic amplification factors for single mass crossing multi-span continuous beam.

$$
\gamma_{i}=0.05, \quad \alpha_{k}=0.1
$$

\subsection{Effect of moving mass to bridge mass ratio $a_{k}$}

Figure 6 demonstrates the difference between a moving mass and a moving force on a two span continuous beam for a range of different mass ratios. At a mass ratio $\alpha_{k}=0.01$ (the moving mass is $1 \%$ of the bridge span mass) we have the very little difference between a moving force and a moving mass problem. If the moving mass is a larger percentage of the mass of a bridge span then there is a significant difference between moving mass and moving force problems. Note that as the mass ratio increase so does the DAF for this case. Review paper [4] suggest that for road traffic the effect of mass ratio is difficult to detect experimentally. This is theoretically because the non-dimensional speed of road traffic (in the range $0<\Omega<0.2$ ) is such that the effect of mass ratios is small on the graph Figure 6.

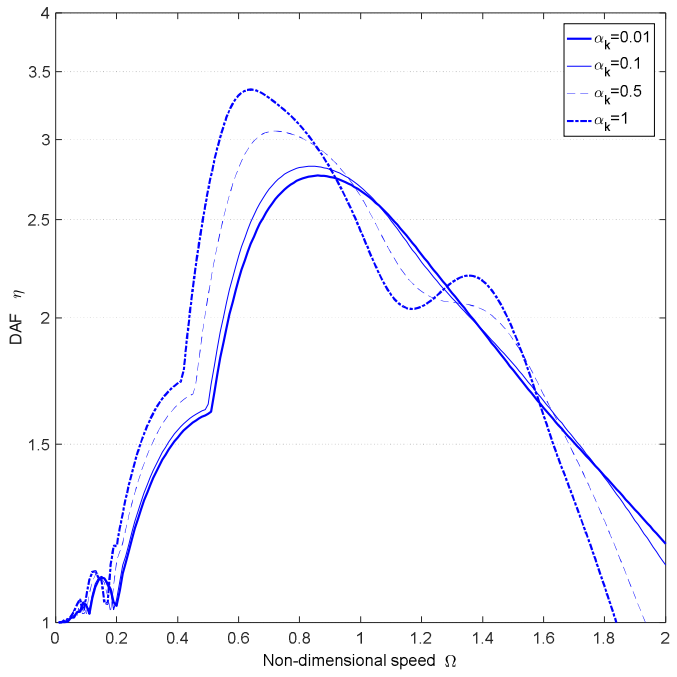

Figure 6, Influence of mass ratio (of moving load) on dynamic amplification factor for

$$
\gamma_{i}=0.05, \quad n=2
$$




\subsection{A set of equispaced moving mass on multi-span beam}

Figure 7 displays the variation of the dynamic application factor vs non-dimensional train speed $\Omega$ and mass ('wheel load') spacing for a train of 9 masses. In the case where $s_{k}=0$ we have the single point mass situation described in the previous section. While when $s_{k}=0.25$ the train length equals $2 L$. The mass ratio of each mass is $0.2 / 9$ which results in a total mass ratio for the train of 0.2 .

We observe that the maximum DAF is approximately equal to the single wheelset mass/load case ( $s_{k}=0$ ). Although the speed at which this maximum occurs is dependent on the spacing length $s_{k}$. As $s_{k}$ increases we see that the speed at which this maximum occurs is higher. For conventional high speed trains this is very beneficial as it pushes this 'resonant' maximum further away from the operating speed. For the case of a Hyperloop system this effect is neither beneficial nor adverse as the operating speed range is so much larger than conventional high speed trains.
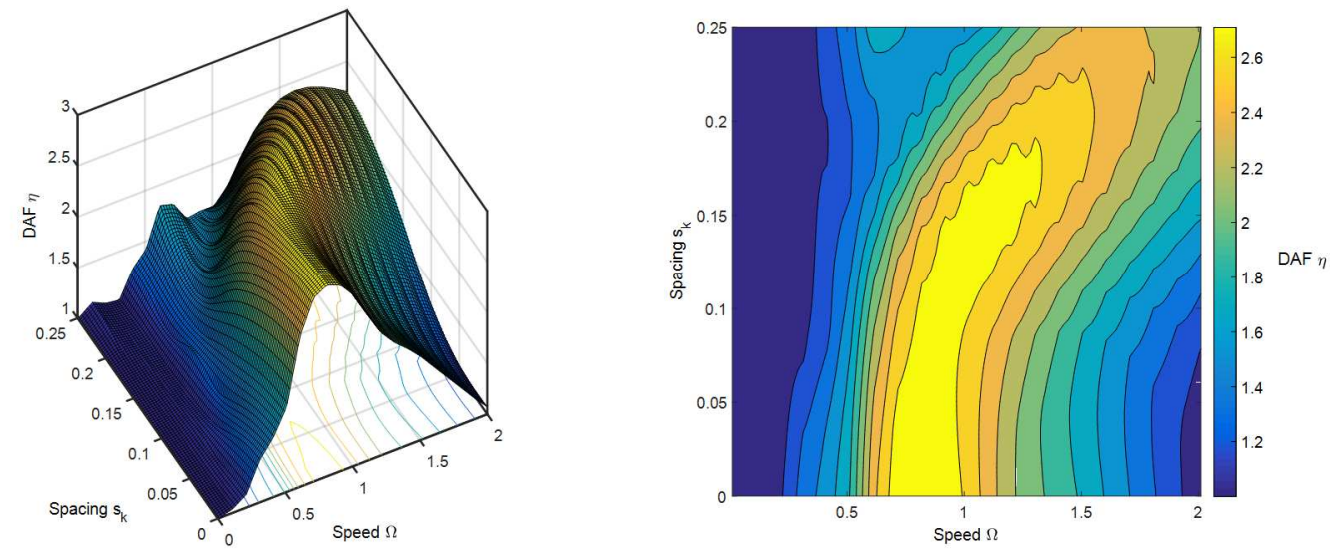

Figure 7, Variation of dynamic amplification factor vs non-dimensional speed and spacing of masses. For case of 2 span continuous beam subjected to a train of 9 equidistance masses

$$
\gamma_{i}=0.05, \quad a_{k}=0.2 / 9
$$

\section{Conclusions}

The proposed speed of Hyperloop/Transpod trains is such that it can introduce significant dynamic amplification due to the travelling masses/loads of the train. This factor, coupled with the suggested continuous beam structure, can result in a very large induced dynamic amplification factor. The maximum value of the DAF for these UHS trains could be as high as the classical resonant amplification of $\eta=1 / 2 \gamma$. This value is so large that either the tubes would have to be very much stiffer than conventional design would suggest or that various vibration absorbing/mitigation devices would have to be included. The analysis also points out that limiting the continuous beam to only a few spans would be dynamically very favourable. However, this may introduce design problems associated with maintaining the vacuum within the tube.

The current code based design regulations/recommendations, world-wide, are insufficient for the design of such systems. 
This paper highlights the need to consider again the dynamics of a set of travelling masses. When the conceptual design of the train carriages, bridge spans, support arrangement are defined, more explicitly, then appropriate analyses of the type proposed in this paper can be undertaken to determine the optimal bridge section design. Given the extremely large dynamic amplification it may be necessary to significantly stiffen the bridge spans and introduce large damping in the form of viscous and tuned mass dampers. The flexibility of the piers and the dynamics of a pair of parallel tubes are not considered in this paper. These are areas for future research as are the interactions between this UHS train-bridge dynamics and wind/earthquake loading.

\section{List of Captions}

Figure 1, initial conceptual design of prototype Hyperloop one.

Figure 2, a train composed of set of $k$ moving point masses traveling across an $n$ span continuous beam

Figure 3, (a) variation of fundamental bridge frequency vs span length (b) variation of non-dimensional velocity $\Omega$ with dimensional span and train speed for Railway Bridges.

Figure 4, (a) normalised midspan deflection (quasi-static, dynamic) vs normalised time (b) dynamic amplification factor (DAF) vs normalised time.

Figure 5, dynamic amplification factors for single mass crossing multi-span continuous beam. $\gamma_{i}=0.05, \quad \alpha_{k}=0.1$

Figure 6, influence of mass ratio (of moving load) on dynamic amplification factor for $\gamma_{i}=0.05, \quad n=2$

Figure 7, variation of dynamic amplification factor vs non-dimensional speed and spacing of masses. For case of 2 span continuous beam subjected to a train of 9 equidistance masses $\gamma_{i}=0.05, a_{k}=0.2 / 9$ 


\section{References}

[1] Musk, E., Hyperloop alpha. Hyperloop Alpha Design Proposal, SpaceX.(Online Article). URL http://www.spacex.com/sites/spacex/files/hyperloop alpha.pdf (last accessed 11/5/2017). 2013

[2] Hyperloop Design Report, Makers UPV Team, Universitat Polit’ecnica de Val’encia, Valencia January 2016.

[3] R. Janzen, "TransPod Ultra-High-Speed Tube Transportation: Dynamics of Vehicles and Infrastructure," Procedia Engineering, vol. 199, no. Supplement C, pp. 8-17, 2017/01/01/2017.

[4] Network Rail, NR/GN/CIV/025 Issue 3, Guidance Note - The Structural Assessment of Underbridges, in, 2006.

[5] Parke G., Hewson. N., ICE Manual of Bridge Engineering, 2nd edition Thomas Telford publishing, 2008

[6] L. Canning, M.M. Kashani, Assessment of U-type wrought iron railway bridges., Proceedings of the Institution of Civil Engineers-Engineering History and Heritage, 169 (2016) 58-67.

[7] B. Standard, EN 1991-2: Eurocode 1: Actions on structures in: Part 2: Traffic loads on bridges., 2003.

[8] S.P. Timoshenko, On the forced vibrations of bridges, Philosophical Magazine Series 6, 43 (1922) 1018-1019.

[9] L. Frýba, Vibration of Solids and Structures under Moving Loads. Groningen, Noordhoff International Publishing Cy. 1972. 511 S., Dfl. 91.35, ÖS 39,75, ZAMM - Journal of Applied Mathematics and Mechanics / Zeitschrift für Angewandte Mathematik und Mechanik, 59 (1979) 489489.

[10] M. Olsson, On the fundamental moving load problem, J. Sound Vibr., 145 (1991) 299-307.

[11] P. Paultre, O. Chaallal, J. Proulx, Bridge dynamics and dynamic amplification factors - a review of analytical and experimental findings, Canadian Journal of Civil Engineering, 19 (1992) 260-278.

[12] M.F. Green, D. Cebon, Dynamic Response of Highway Bridges to Heavy Vehicle Loads: Theory and Experimental Validation, J. Sound Vibr., 170 (1994) 51-78.

[13] P. Salcher, C. Adam, Modeling of dynamic train-bridge interaction in high-speed railways, Acta Mechanica, 226 (2015) 2473-2495.

[14] H. Xia, N. Zhang, G. De Roeck, Dynamic analysis of high speed railway bridge under articulated trains, Computers \& Structures, 81 (2003) 2467-2478.

[15] Y.-B. Yang, J.-D. Yau, L.-C. Hsu, Vibration of simple beams due to trains moving at high speeds, Engineering Structures, 19 (1997) 936-944.

[16] K. Liu, E. Reynders, G. De Roeck, G. Lombaert, Experimental and numerical analysis of a composite bridge for high-speed trains, J. Sound Vibr., 320 (2009) 201-220.

[17] N. Zhang, H. Xia, W. Guo, Vehicle-bridge interaction analysis under high-speed trains, J. Sound Vibr., 309 (2008) 407-425.

[18] D.A. Michal, Matrix and Tensor Calculus: With Applications to Mechanics, Elasticity and Aeronautics, Dover publishing Inc, 2008.

[19] J. M. T. Thompson and G. W. Hunt, Elastic instability phenomena, Great Britain: John Wiley and Sons, London, 1984

[20] G.P. Tilly, Dynamic behaviour of concrete structures. , in: In Developments in civil engineering, Vol. 13, Report of the Rilem 65MDB Committee, 1986.

[21] Ray W. Clough, J. Penzien, Dynamics of Structures, Third Edition ed., Computers \& Structures, Inc., 1995. 